\title{
Interactive Deformable Registration Visualization and Analysis of 4D Computed Tomography
}

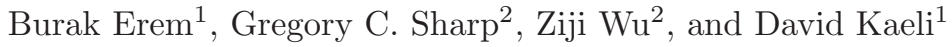 \\ ${ }^{1}$ Department of Electrical and Computer Engineering, Northeastern University \\ Boston, MA 02115 \\ ${ }^{2}$ Department of Radiation Oncology, Massachusetts General Hospital \\ Boston, MA 02114
}

\begin{abstract}
This paper presents a new interactive method for analyzing 4D Computed Tomography (4DCT) datasets for patient care and research. Deformable registration algorithms are commonly used to observe the trajectories of individual voxels from one respiratory phase to another. Our system provides users with the capability to visualize these trajectories while simultaneously rendering anatomical volumes, which can greatly improve the accuracy of deformable registration.
\end{abstract}

\section{Introduction}

Radiation therapy is a method of treating patients with various types of cancerous tumors. The goal of the treatment is to kill cancerous cells by exposing them to radiation but, when exposed, enough radiation will kill healthy tissue as well. This becomes more of a concern for a physician planning a patient's treatment when the location of the tumor moves significantly due to cardiac activity or respiration, often leading to lower treatment success rates. For this reason, much research in this area focuses on minimizing exposure of healthy tissue to radiation while maximizing the coverage of the intended target. With a better understanding of internal anatomical motion, physicians can improve the accuracy and efficiency of the treatment of their patients.

One attempt at characterizing such motion is by using a deformable registration algorithm on $4 \mathrm{DCT}$ data to map, voxel by voxel, movement from one respiratory phase to another. Based on splines, this model of voxel trajectories can have undesirable results if the algorithm's parameters are not appropriately set. Furthermore, it can be difficult to determine what the proper parameters should be without some visual feedback and experimentation.

This paper introduces several new ideas for medical visualization that can help address this issue. For the visualization of anatomical motion in 4DCT image sets, we present the ability to display point trajectories. Specifically, we have developed a toolset that can simultaneously visualize vector fields and anatomy, provides interactive browsing of point trajectories, and allows for the improved identification of current trajectory position using node color. We also describe 
some additional interactive capabilities of our work, such as editing of deformation fields which can enable automatic and interactive registration.

\section{Background}

The analysis of anatomical trajectories relies heavily on mathematics, but also heavily relies on a visual interpretation of the data. Thus, because simultaneous mathematical and visual analysis is important, in this section we will discuss the registration and visualization background that has influenced the work presented in this paper.

\subsection{Deformable Registration}

Image registration is a process to determine a transformation that can relate the position of features in one image with the position of the corresponding feature in another image. Spline-based free-form registration is capable of modeling a wide variety of deformations and also, by definition, ensures a smooth deformation field. A deformation field is represented as a weighted sum of spline basis functions, of which B-splines are one of the most widely used. In the B-spline transformation model [1], the deformation vectors are computed using B-spline interpolation from the deformation values of points located in a coarse grid, which is usually referred to as the B-spline grid. The parameter space of the B-spline deformation is composed by the set of all the deformations associated with the nodes of the B-spline grid. A cubic B-spline in matrix form is:

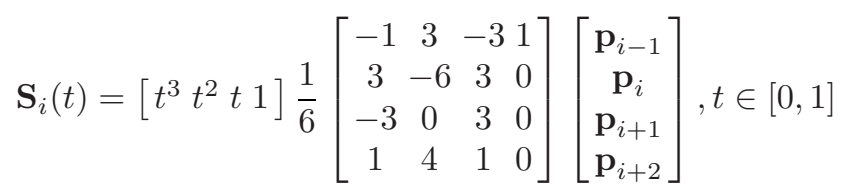

where $\mathbf{p}_{j}$ are the control points. Note that B-splines have a finite support region. Thus changing the weight or contribution of each basis function affects only a specific portion of the overall deformation. By increasing the resolution of the B-spline grid, more complex and localized deformations can be modeled.

An alternative to the B-spline deformation model is landmark-based splines, typically implemented using thin-plate splines [2] or other radial basis functions. In this approach, a set of landmark correspondences matches is formed between points in a pair of images. The displacements of the correspondences are used to define a deformation map, which smoothly interpolates or approximates the point pairs. One approach of particular interest is radial basis functions that have finite support, such as the Wendland functions [3]. Because these functions only deform a small region of the image, the deformations can be quickly computed and updated for interactive applications. Given $N$ points, located at $x_{i}$ and displaced by an amount $\lambda_{i}$, the deformation $\boldsymbol{\nu}$ at location $x$ is given as:

$$
\boldsymbol{\nu}(x)=\sum_{i=1}^{N} \lambda_{i} \phi\left(\left|x-x_{i}\right|\right),
$$


where $\phi$ is an appropriate Wendland function, such as:

$$
\phi(r)= \begin{cases}\left(1-\frac{r}{\sigma}\right)^{2} & r \leq \sigma \\ 0 & \text { otherwise }\end{cases}
$$

The variable $\sigma$ controls the width of the adjustment, usually on the order of one to two centimeters for human anatomy. Several of these Wendland functions are used together to form a complete vector field, which defines the motion of organs of the anatomy.

\subsection{Volume Visualization}

Volume visualization is a way of viewing the structure of the anatomy in 3D. The main goal of volume visualization is to represent higher dimensional data on a two-dimensional computer screen for visual inspection. In this case, we use visualizations of the same 4DCT datasets which we have used for deformable registration calculations, providing a superimposed anatomical frame of reference for analysis.

While it is common to see 3D renderings of human anatomy in this field, it is important to note that there are several methods of obtaining these visualizations with important distinctions between them. We separate these into two categories: 1) direct volume rendering and 2) explicit volume rendering. With explicit volume rendering, the boundaries of the structure which are being visualized are explicitly defined, calculated, and then projected onto the $2 \mathrm{D}$ viewing plane of the user. On the other hand, direct volume rendering only calculates the surfaces which will be projected onto the $2 \mathrm{D}$ viewing plane, making it a faster alternative.

We chose to work with direct volume rendering in our analysis because of its inherent speed advantage. We note that there is no loss of information from the user's perspective with this method, especially from the standpoint of analyzing and editing deformable registration parameters. It is because the renderings act as a reference for visual comparison to the independent registration calculations that explicit surfaces are not necessary.

\subsection{SCIRun}

Developed by the Scientific Computing and Imaging (SCI) Institute at the University of Utah, SCIRun is a problem solving environment designed to allow researchers the freedom to build programs to perform various scientific computing tasks [4]. In our particular application, a dataflow network of modules already existed that allowed us to do direct volume rendering. The network is a simplified version of the SCIRun PowerApp called BioImage [5]. Enhancements were made to that network to allow for visualization of 4DCT datasets and point paths by cycling through them one phase at a time. Building on the existing tools, we can provide for more efficient and interactive ways of analyzing tumor motion. 
The viewing window, the central module to which almost all dataflow eventually leads, is especially useful for our application. This graphical viewport allows navigation of the 3D environment in which we work by zooming, panning, and rotating. Furthermore, the viewing window passes back event callbacks to certain classes of objects that allow module developers to make interactive, draggable, clickable tools. However, movement of such tools is limited to the viewing plane. Thus, by rotating the viewing plane, one is able to change the directions of motion of the interactive tools.

\section{Methodology}

\subsection{Viewing Trajectory Loops}

To represent a 4D trajectory in a 3D graphical environment, we have developed a cursor that displays the path of movement of a single voxel over time. A user can move the cursor by clicking and dragging it in a motion plane parallel to the viewing plane. At its new location, the cursor displays the trajectory of the voxel at that point by showing a line path. The direction and magnitude of the motion during each time phase are indicated by a color transition from blue to red.

All trajectories start and end at the same shades of blue and red, but may display less of certain intermediate shades due to very low magnitude movements during those time phases. This can be very useful when comparing two trajectories of similar shape, but very different color patterns, indicating that despite having followed a similarly shaped path, each voxel followed the path at a different speed.

\subsection{Editing Point Paths}

Once a user has identified a region of interest using our tool, they can then explore the region in greater detail. Instead of displaying a line path, this tool displays several cursors to convey similar information without using lines. To prevent confusion about the order, the module connects to the same tool that allows the user to select the 4DCT phase currently being viewed, and then highlights the corresponding cursor with a different color. At each respiratory phase, the path of a voxel can be followed both through this tool and a volume visualization simultaneously.

If it is observed that the trajectory and the visualization do not agree, the user has the option of editing the trajectory by moving the cursors. It should be noted that this will not modify the 4DCT data itself, but only supplement the output of the registration algorithm. Also, moving the cursor will not only effect the voxel whose trajectory is being viewed, but will also have an attenuated effect on the surrounding area. To view the extent of this effect, the user can use several of the previously described tools to view the updated trajectory loops. 

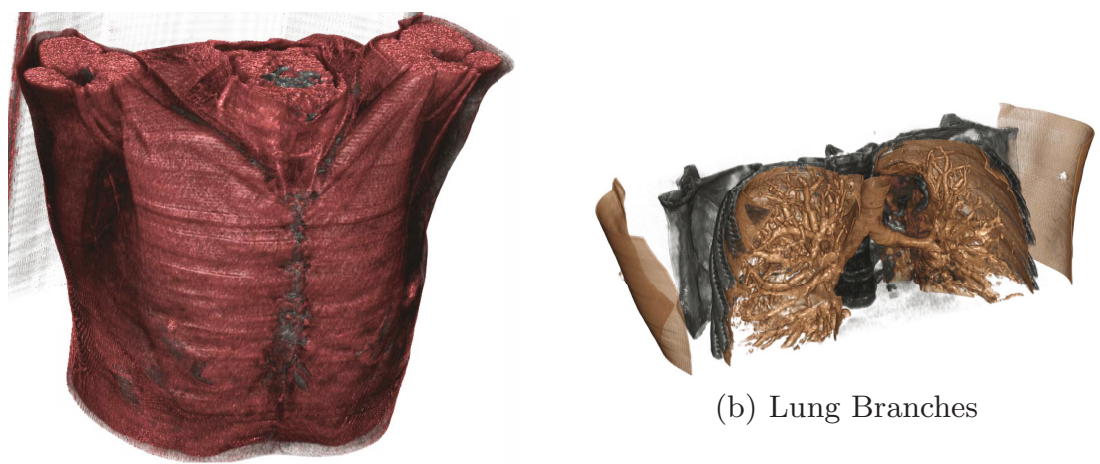

(b) Lung Branches

(a) Muscle

Fig. 1. (a) Visualization of bone and muscle. Although it is possible to analyze trajectories within this type of visualization, or (b) one showing the branches of the lungs, we provide examples showing only bony anatomy for visual clarity.

\section{Results}

The visual nature of these tools provides a definite improvement in the way tumor motion analysis is performed. The user has a rich set of visualization capabilities using our system; volume rendering of 4DCT datasets is capable of showing many different kinds of tissue. Figure 1 shows two examples of different kinds of tissue that can be visualized. In Figure 1(a) we show how muscle and bone can be displayed simultaneously and that our visualization tools are not strictly limited to bone. Figure 1(b) shows branches of a lung, an important kind of tissue whose motion is important to understand in order to treat tumors located within. However, for the rest of the figures, we use renderings of bony anatomy only to avoid cluttering the view of our tools. It should be noted that this is less of a concern when viewing them together in an interactive environment.

The trajectory loop tool's purpose is to facilitate rapid analysis of trajectories within the visual environment. We are able to run the trajectory loop at every position the cursor has been (see Figure 2), showing a trail of several loops that have been visualized. In this figure, the tool was used to analyze the extent to which the registration algorithm detected motion at various spatial locations within the lung. As expected, movements became smaller as the cursor was used to inspect areas closer to bone. On the other hand, trajectory loops closer to the lower lung showed significant motion.

If unsatisfied with analysis of the trajectories when compared to the visualization, the user can make adjustments within this environment to improve the registration. Figure 3 shows the path editing tool, where each of the individual points can be moved independently to adjust the path to the user's specifications. The point that is colored green highlights the current phase of the 4DCT that is being visualized. Thus, if the rest of the anatomy were visible, one could see the voxel to which that specific point path belonged. While Figure 3(a) shows 


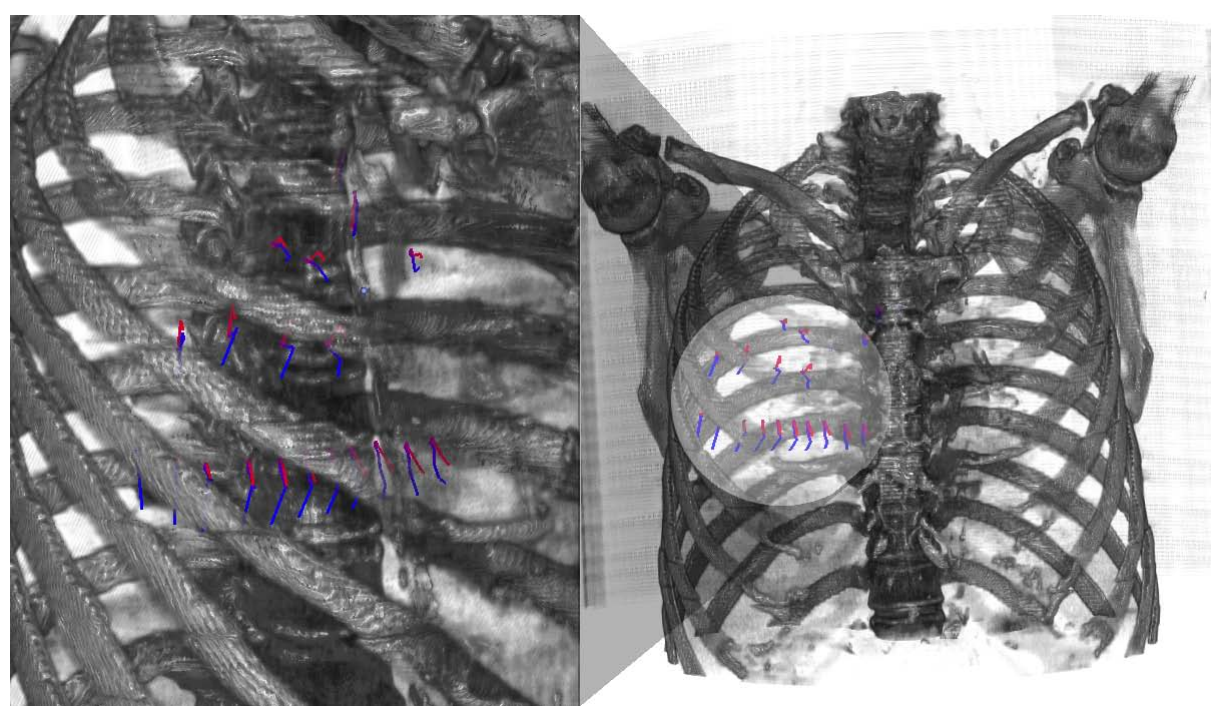

Fig. 2. Viewing several trajectories in the lung while visualizing surrounding bony anatomy (right) and while zoomed in (left). Trajectories are represented as line loops that make a smooth transition from blue to red in even increments across each of the respiratory phases.

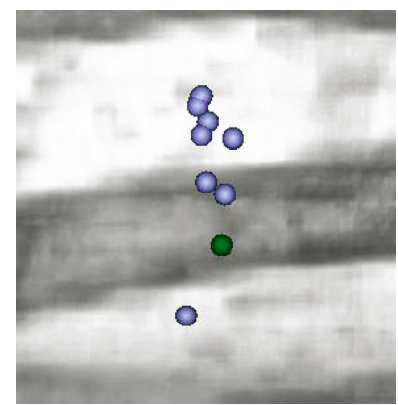

(a) Zoomed In

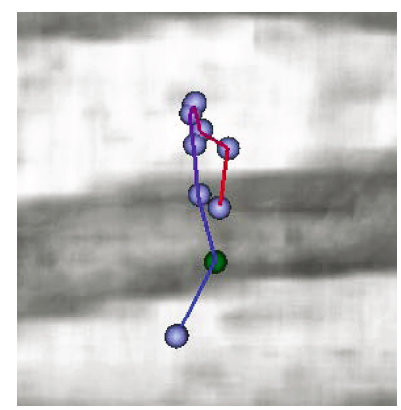

(b) With Loop

Fig. 3. (a) The editing tool shown alone with the current phase highlighted in green and (b) the same editing tool shown with the trajectory loop superimposed to demonstrate the relationship between the two tools. The point highlighted in green is edited while the others remain fixed to prevent accidental changes.

the editing tool alone, Figure 3(b) shows the trajectory loop tool and the path editing tool when used at the same point. This may not normally be a desired way to edit a path, but in this case it serves to illustrate the relationship between the two tools. Each has its own purpose for different intended uses, but this demonstrates that both represent the same registration information. 


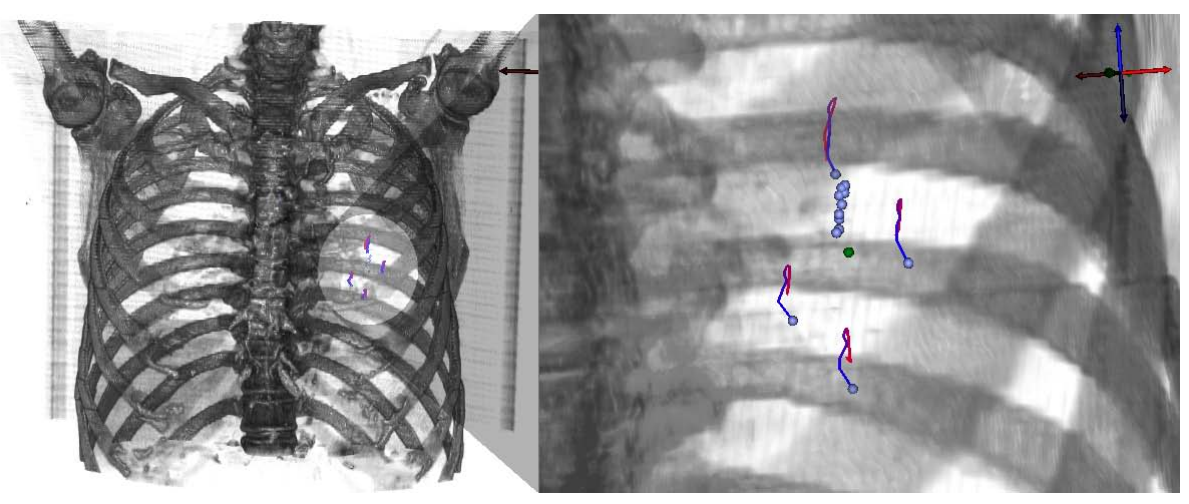

Fig. 4. A zoomed out (left) and more detailed (right) perspective of editing a point path while observing changes using the trajectory loop tool

When changes are made to the point path and are committed, the tool appends modifications to the previous registration results and refreshes the visualization. Changing the visible path affects the surrounding paths as well, in a way similar to how smudging tools work in image editing software. The effect of this range of influence can be seen by using the path editing tool and several trajectory loop tools simultaneously, as shown in Figure 4. This kind of visual feedback allows a user to avoid impacting surrounding trajectories that move independently of the one being directly edited. Similarly, if those kinds of changes are desired for an entire object at once, the tool can provide for this as well.

\section{Future Work}

Our future efforts with this work will focus on three major areas of advancement: visualization, interactivity, and pattern matching. In the area of visualization, we will use other visual queues such as color or line thickness to highlight additional attributes of motion such as velocity and regularity. Further, as movement is not fully characterized by trajectories alone, we will include visualizations of tissue expansion and contraction to enable new analyses.

To improve interactivity, we will explore more efficient means of marking regions whose trajectories have been deemed unreliable. One approach may be to paint a region in $3 \mathrm{D}$ and recompute the registration for that region alone. Additionally, we plan on learning registration algorithm parameters from the way researchers use these interactive tools.

Lastly, we will explore motion correlation measurement and analysis between different regions of the anatomy using a combination of the visualization and interactive tool creation capabilities that we have shown and the pattern matching mentioned above. 


\section{Conclusion}

In this paper we have introduced a set of tools that allow researchers characterizing anatomical motion to visualize and edit deformation fields in a 3D environment. While it is clear that deformable registration is a valuable research tool for the area of anatomical motion research, we have been able to improve the usefulness of the algorithm by providing an intuitive interface that displays information more efficiently, encourages more integration with other forms of visualization, and provides a way of interacting with the data to make changes to the model in the same environment. We have also described some of the future directions for this project.

\section{Acknowledgments}

This work was supported in part by Gordon-CenSSIS, the Bernard M. Gordon Center for Subsurface Sensing and Imaging Systems, under the Engineering Research Centers Program of the National Science Foundation (Award Number EEC-9986821). This work was made possible in part by software from the NIH/NCRR Center for Integrative Biomedical Computing, P41-RR12553-07.

\section{References}

1. Rueckert, D., Sonoda, L.I., Hayes, C., Hill, D.L.G., Leach, M.O., Hawkes, D.J.: Nonrigid registration using free-form deformations: Application to breast MR images. IEEE Trans. on Med. Imag. 18(8), 712-721 (1999)

2. Bookstein, F.L.: Principal warps: Thin-plate splines and the decomposition of deformations. IEEE Trans. Pattern Anal. Mach. Intell. 11(6), 567-585 (1989)

3. Fornefett, M., Rohr, K., Stiehl, H.S.: Radial basis functions with compact support for elastic registration of medical images. Image and Vision Computing 19(1-2), 87-96 (2001)

4. SCIRun.: A Scientific Computing Problem Solving Environment, Scientific Computing and Imaging Institute (SCI)

5. BioImage.: Volumetric Image Analysis and Visualization. Scientific Computing and Imaging Institute (SCI) 